\title{
SHORT-TERM OUTCOME OF PATIENTS WITH HEPATOPANCREATOBILIARY MALIGNANCIES TREATED IN A MULTIDISCIPLINARY HEPATOBILIARY UNIT IN A TERTIARY REFERRAL CENTRE
}

\author{
Muhammad F. Shah, Raza Sayyed, Abid Q. Qazi, Shahid Khattak, Aamir A. Syed, Faisal Hanif \\ Department of Surgical Oncology, Shaukat Khanum Memorial Cancer Hospital and Research Center, Lahore, \\ Pakistan
}

Received: 6 January 2016 / Accepted: 1 April 2016

\begin{abstract}
Purpose: Specialisation in hepatopancreatobiliary (HPB) surgery has evolved over the past few decades based on better understanding of the biology and the behaviour of the diseases. In this review from a specialised HPB service, we aim to provide an overview of the HPB oncological cases managed in a tertiary care cancer hospital.

Methods: All cases of HPB malignancies treated in our hospital between October 2014 and September 2015 were included in the study. Clinical findings, operative details and short-term post-operative outcomes were assessed from a prospectively managed database.

Results: A total of 65 oncological procedures were performed over 1 year. These included 23 hepatic resections, 27 pancreaticoduodenectomies, 5 radical surgeries for gallbladder cancer, 2 distal pancreatectomy and 8 nephrectomies with exploration of the inferior vena cava. One patient successfully underwent Associated Liver Partition with Portal vein ligation and staged hepatectomy (ALPPS Procedure), which to our knowledge was the first ALPPS procedure carried out in Pakistan.
\end{abstract}

Conclusion: The short-term experience of patients managed in specialised HPB unit has shown good outcomes. There is a need for establishing HPB units in most tertiary care hospitals in the country.

Key words: Associated liver partition with portal vein ligation and staged hepatectomy procedure, hepatic resections, hepatopancreatobiliary surgery, pancreaticoduodenectomy

\section{Introduction}

Specialisation in hepatopancreatobiliary (HPB) surgery has evolved over the past few decades based on better understanding of the biology, the behaviour of the diseases, improvements in diagnostic modalities, development of a highly specialised skills set in the operating room and evolution of multidisciplinary care of diseases, especially cancers. ${ }^{[1,2]}$ In addition, the outcomes of complex operations are strongly linked to the institution as well as the surgeon's operative volumes. ${ }^{[3-6]}$ Mortality and operative outcomes for HPB cancers have, in particular, improved dramatically as shown by

Correspondence: Dr. Faisal Hanif, Department of Surgical

Oncology, Shaukat Khanum Memorial Cancer Hospital and Research

Centre, Lahore, Pakistan.

Email: faisalhanif@skm.org.pk published experience from specialised centres all over the world. ${ }^{[7,8]}$

The age-standardised incidences for cancers of the liver, gallbladder and pancreas are 3.6, 1.5 and 0.5 per 100,000 population. ${ }^{[9]}$ This reflects a significant burden of cancer in Pakistani population with tumours that can be potentially curable with timely availability of expertise in their complex surgical and oncological management. ${ }^{[10-12]}$ In this review from a specialised HPB service, we aim to provide an overview of the HPB oncological cases managed in a tertiary care cancer hospital.

\section{Methods}

All cases of HPB malignancies treated in our hospital between October 2014 and September 2015 were included 
in the study. The HPB unit also provided assistance to the urological and paediatric surgeons with resection of complex renal tumours, especially, when there was inferior vena cava (IVC), liver or pancreatic involvement. Patient's demographics, clinical findings, operative details and short-term post-operative outcomes were assessed from a prospectively managed database.

\section{Results}

A total of 65 oncological procedures were performed over 1 year. These included 23 hepatic resections, 27 pancreaticoduodenectomies, 5 radical surgeries for gallbladder cancer, 2 distal pancreatectomy and 8 nephrectomies with exploration of the IVC. General emergency procedures and staging laparoscopies were excluded from analysis.

Liver resections were performed in 23 patients. The types of liver tumours treated are described in Figure 1. Anatomic segmental resections were performed in 10 patients, while 8 patients underwent sectionectomies. Three patients underwent right hemihepatectomy, while extended right hemihepatectomy was performed in one patient. One patient successfully underwent Associated Liver Partition with Portal vein ligation and Staged hepatectomy (ALPPS Procedure), which to our knowledge was the first ALPPS procedure carried out in Pakistan. Median age was 52 years (range 1-71 years). There were 15 male and 8 female patients in this group. Median intensive care unit (ICU) stay was 34 h. Median hospital stay was 8 days (range 4-9 days). Postoperatively, two patients had perihepatic haematoma, one had wound infection and one had myocardial infarction. There was no mortality.

Twenty-seven patients successfully underwent pancreaticoduodenectomy and two patients underwent distal pancreatectomy. In six patients, tumours were found inoperable on trial of dissection. In one patient, portal vein resection and reconstruction were performed. Two patients had previously undergone palliative bypass surgery in outside tertiary care hospitals for pancreatic head cancer. Both of these patients underwent successful pancreaticoduodenectomy in our hospital.

In the pancreaticoduodenectomy group, median age was 51 years with 17 male and 10 female patients.
Median tumour size was $28 \mathrm{~mm}$ (range 15-95 $\mathrm{mm}$ ). The most common presenting symptom was jaundice $(n=22,81 \%)$ followed by pain $(n=5,18.5 \%)$ and weight loss $(n=4,14.8 \%)$. Periampullary tumours constituted ( $n=13,48.4 \%$ ), followed by carcinoma of head of pancreas $(n=9,32.3 \%)$ and duodenal carcinoma $(n=5,19.3 \%)$. On histopathology, there were 24 adenocarcinomas, 2 neuroendocrine tumours and one gastrointestinal stromal tumour. With the exception of one patient, all patients had R0 resection. One patient had R1 resection with positive resection margin at the uncinate process. Aberrant hepatic vascular anatomy was found in $40 \%$ of the patients undergoing pancreaticoduodenectomy. Post-operative pancreatic fistula developed in $18 \%$ $(n=5)$ of patients. These were International Study Group in Pancreatic Surgery Grade A except for one patient who required percutaneous drainage. Delayed gastric emptying was seen in $18 \%(n=5)$ of patients. One patient had upper gastrointestinal haemorrhage managed endoscopically and one developed intra-abdominal abscess that required image-guided percutaneous drainage. One patient died on post-operative day 7 due to cardiac event.

The HPB service also provided complex vascular cover to adult and paediatric urology service in the hospital. This allowed successful surgery for 8 patients with tumours of the kidneys or adrenal glands requiring management of the IVC. These included three cases of renal cell cancer, 3 Wilm's tumour, 1 adrenal tumour and 1 clear cell sarcoma of the kidney. IVC thrombectomy with lateral repair was performed in six patients, and one patient had segmental resection of IVC and anastomosis. One patient required en bloc resection of the right adrenal and the right kidney in conjunction with a right hemihepatectomy [Figure 2]. There was no mortality in this group.

\section{Discussion}

We report the 1 full year outcome of managing patient with HPB malignancies in a newly established HPB service in a tertiary care cancer hospital. A large number of complex resections were performed with morbidity and mortality comparable to published literature. Of note is successful surgery of locally advanced cancers which were deemed inoperable and had undergone palliative surgery in other centres. In addition, patients with locally advanced tumours who were deemed unsuitable for surgical 


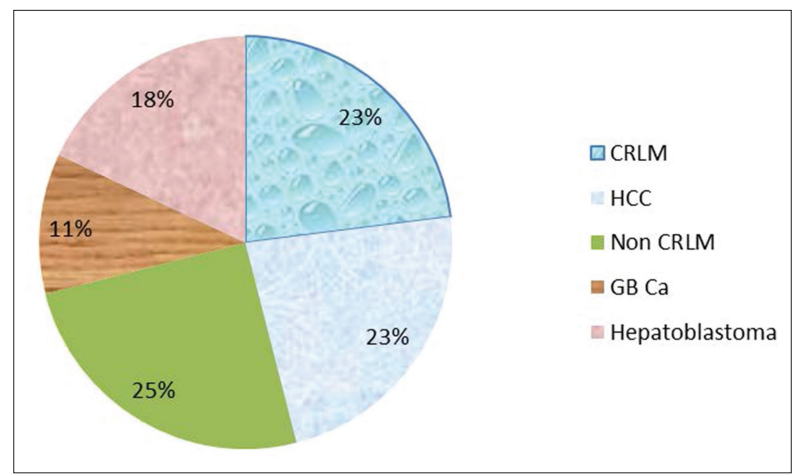

Figure 1: Indications for liver resection in our cohort of patients (colorectal liver metastasis [CRLM], hepatocellular carcinoma [HCC], non-CRLM, gallbladder carcinoma [GB Ca])

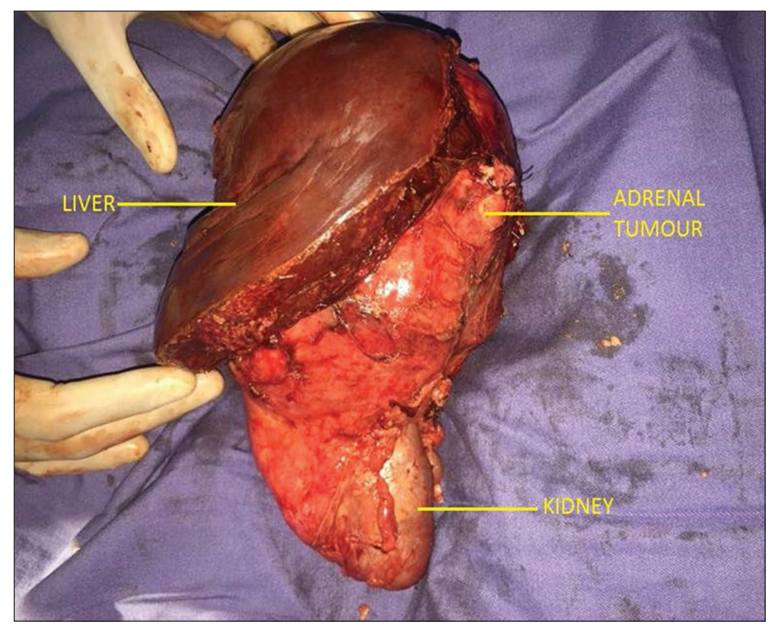

Figure 2: En bloc resection of the right lobe of the liver with right adrenal tumour and kidney

resection and denied surgery in other centres received successful surgical management for liver, pancreatic and renal tumours with multivisceral involvement.

Pancreatic and liver resections for tumours have been associated with high morbidity and mortality. ${ }^{[1,2]}$ However, with a multidisciplinary approach advances in surgical techniques, better understanding of the disease process, oncological management and post-operative care, one can achieve excellent outcomes after the complex resections for HPB tumours..$^{[7,8]}$ Our study supports the view that the patients with HPB malignancies treated in specialised HPB units are likely to have a better outcome. ${ }^{[3,6]}$

The HPB service at Shaukat Khanum Memorial Cancer Hospital and Research Centre was established to streamline the care of patients with HPB malignancies. All patients are staged with 64-Slice computed tomography scan with additional magnetic resonance imaging and endoscopic ultrasound if required as per discussion in the multidisciplinary team meeting comprising surgeons, gastroenterologists, radiologists and oncologists. The operating rooms have state-of-the art equipment including Energy devices and Cavitron Ultrasonic Suction Aspirator device. Post-operative care for these patients includes a well-equipped ICU as well as a surgical extended care unit for patients stepping down from ICU.

There is a countrywide need to develop specialised HPB units to achieve good results from these complex procedures. This may be achieved by obtaining expertise and training in HPB surgery as well as developing a multidisciplinary team management plan for these patients. In addition, fellowship training programs in HPB surgery should be developed to increase the number of trained HPB professionals.

\section{Conclusion}

HPB malignancies pose many challenges to treatment that requires a multidisciplinary approach in addition to advanced diagnostic, therapeutic and technical expertise. The short-term experience of patients managed in specialised HPB unit has shown good outcomes. There is a need for establishing HPB units in most tertiary care hospitals in the country.

\section{Conflict of Interest}

The authors declare that they have no conflict of interest.

\section{References}

1. Bismuth H. Surgical specialization. Br J Surg 2013;100:S43-4.

2. Carter DC. The specialty of hepatobiliary surgery. Br J Surg 1987;74:871-2.

3. Csikesz NG, Simons JP, Tseng JF, et al. Surgical specialization and operative mortality in hepato-pancreaticobiliary (HPB) Surgery. J Gastrointest Surg 2008;12:1534-9.

4. Shrikhande SV. Whipple resection: The need for specialization, standardization and centralization. South Asian J Cancer 2013;2:158-9.

5. Eppsteiner RW, Csikesz NG, Simons JP, et al. High Volume and outcome after liver resection: Surgeon or center? J 
Gastrointest Surg 2008;12:1709-16.

6. Shrikhande SV, Barreto SG, Somashekar BA, et al. Evolution of pancreatoduodenectomy in a tertiary cancer center in India: Improved results from service reconfiguration. Pancreatology 2013;13:63-71.

7. Cameron JL, He J. Two thousand consecutive pancreaticoduodenectomies. JAm Coll Surg 2015;220:530-6.

8. Kamiyama T, Nakanishi K, Yokoo H, et al. Perioperative management of hepatic resection toward zero mortality and morbidity: Analysis of 793 consecutive cases in a single institution. J Am Coll Surg 2010;211:443-9.

9. GLOBOCAN 2012 Estimated Cancer Incidence, Mortality and Prevalence Worldwide IARC. Lyon France: (IARC)
Section of Cancer Surveillance; 2012. Available from: http:// www globocan.iarc.fr/Pages/fact_sheets_populat ion.aspx. [Last accessed on 2016 Jan 04].

10. Wagner M, Redaelli C, Lietz M, et al. Curative resection is the single most important factor determining outcome in patients with pancreatic adenocarcinoma. Br J Surg 2004;91:586-94.

11. Kiran RP, Pokala N, Dudrick SJ. Incidence pattern and survival for gallbladder cancer over three decades: An analysis of 10301 patients. Ann Surg Oncol 2007;14:827-32.

12. Riall TS, Cameron JL, Lillemoe KD, et al. Resected periampullary adenocarcinoma: 5-year survivors and their 6-to 10-year follow-up. Surgery 2006;140:764-72. 